\title{
On Singularities of the Heat Flow for Harmonic maps from Surfaces into Spheres
}

\author{
JIE QING
}

\begin{abstract}
In this paper we prove that there is no unaccounted energy loss for blow-ups of solutions of the heat equation for harmonic maps from surfaces into spheres. Any energy drop corresponds precisely to the production of bubbles, that is, nontrivial harmonic maps from 2-sphere into the target
\end{abstract}

\section{Introduction.}

In this paper we shall study the local behavior of solutions of the heat equation for harmonic maps from surfaces into spheres near singular points. About ten years ago, Michael Struwe [17] proved that global solutions of the heat equation for harmonic maps from surfaces exist and are regular except possibly at finitely many isolated points. Recently K.C.Chang, W.Ding and R.Ye [6] showed that these singular points indeed exist in some cases. Naturally then, one would like to know what happens to the solutions near these singular points. In fact, to get a complete description of the set of harmonic maps by Morse theory it might be helpful to know the local behavior of the solutions near singular points, since the solutions of the heat equation for harmonic maps can be naturally interpreted as the trajectories of gradient-like vector fields with respect to the energy functional.

For simplicity, we assume that the domain is just $R^{2}$. The heat equation for harmonic maps is as follows:

$$
\left\{\begin{array}{l}
\partial_{t} u=\Delta u+A(u)(\nabla u, \nabla u) \text { in } R^{2} \\
u(x, 0)=u_{0}(x)
\end{array}\right.
$$

where $A(\cdot)(\cdot, \cdot)$ is the second fundamental form of the target manifold $N$ in some $R^{k}$, and $u_{0}$ is a map in $W^{1,2}\left(R^{2}, N\right)$ with finite energy. By Struwe's results [17] we know, for any $u_{0}$ with finite energy, there exists a global solution

\footnotetext{
${ }^{1}$ Part of this paper was done when the author was supported by NSF grant DMS 9304580.
} 
for (1.1) which is regular except at finitely many points $\left\{\left(x_{j}, T_{j}\right)\right\}_{j=1}^{l}=S$. Let $T$ be any one of $\left\{T_{j}\right\}_{j=1}^{l}$, and let $u(x, T)$ be a weak limit of the solution $u(x, t)$ as $t \rightarrow T$. Then we have

Theorem 1.1. Suppose that $N$ is a sphere and $u(x, t)$ is a solution of (1). Furthermore, assume that $\left(x_{0}, T\right) \in S$. Then there exist finitely many, nonconstant, harmonic maps $\left\{\omega_{k}\right\}_{k=1}^{p}$ from $S^{2}$ into $N$ such that

$$
\lim _{t \rightarrow T} \int_{B_{R}}|\nabla u|^{2}(\cdot, t) d x=\int_{B_{R}}|\nabla u(\cdot, T)|^{2} d x+\sum_{k=1}^{p} E\left(\omega_{k}\right),
$$

for any disk $B_{R}=B_{R}\left(x_{0}\right)$ with $\overline{B_{2 R}\left(x_{0}\right)} \bigcap\left\{x \neq x_{0}:(x, T) \in S\right\}=\Phi$. Moreover, for some sequence $\left\{t_{i}\right\}$ with $\lim _{i \rightarrow \infty} t_{i}=T$ there exist

(a) sequences $\left\{a_{i}^{1}\right\},\left\{a_{i}^{2}\right\}, \cdots\left\{a_{i}^{p}\right\}$ in $R^{2}$ with $\lim _{i \rightarrow \infty} a_{j}^{k}=x_{0}$ for $1 \leq k \leq p$, and

(b) sequences $\left\{\lambda_{i}^{1}\right\},\left\{\lambda_{i}^{2}\right\}, \cdots\left\{\lambda_{i}^{p}\right\}, \lambda_{i}^{k}$ positive for $1 \leq k \leq p$ and any $i$, and $\lim _{i \rightarrow \infty} \lambda_{i}^{k}=0$ for $1 \leq k \leq p$, satisfying, for $j \neq k$,

$$
\frac{\lambda_{i}^{k}}{\lambda_{i}^{j}}+\frac{\lambda_{i}^{j}}{\lambda_{i}^{k}}+\frac{\left|a_{i}^{k}-a_{i}^{j}\right|^{2}}{\lambda_{i}^{k} \lambda_{i}^{j}} \rightarrow \infty, \text { as } i \rightarrow \infty,
$$

and

$$
u\left(x, t_{i}\right)-\sum_{k=1}^{p}\left(\omega_{k}\left(\frac{x-a_{i}^{k}}{\lambda_{i}^{k}}\right)-\omega_{k}(\infty)\right) \rightarrow u(x, T)
$$

strongly in $W^{1,2}\left(B_{R}, R^{k}\right)$, where $\omega_{k}(\infty)$ is the image of $\omega_{k}$ at North pole of $S^{2}$.

Remark 1. (1.2) implies there is no unaccounted energy loss. In other words, the necks connecting bubbles hardly contribute any energy. In fact, this point is one of the main difficulties in the proof. Jost, in the book [11], has proved a similar result for certain min-max values.

Remark 2. (1.4) is only proved for certain sequence $\left\{t_{i}\right\}$, since we do not know whether this configuration of bubbles is unique or not when the sequence varies: This is a very interesting and challenging open problem.

The proof of Theorem 1.1 is indirect. Our strategy is to grab all bubbles in one time. Then by appropriate scaling, turn the problem into that of blowups of a Palais-Smale sequence. For this purpose we prove the following proposition, which may be considered as an improvement of Proposition 5.1 in [17]. 
Proposition 1.2. Suppose that $u_{n} \in W^{1,2}\left(R^{2}, S^{N}\right)$ with $E\left(u_{n}\right) \leq C$, and that $E^{\prime}\left(u_{n}\right) \rightarrow 0$ in $L_{l o c}^{2}\left(R^{2}, R^{K+1}\right)$. Then there may exist

(a) sequences $\left\{a_{n}^{1}\right\},\left\{a_{n}^{2}\right\}, \cdots\left\{a_{n}^{p}\right\}$ in $R^{2}$ with $\lim _{i \rightarrow \infty} a_{n}^{k}=x^{k}$ for $1 \leq k \leq p$, and

(b) sequences $\left\{\lambda_{n}^{1}\right\},\left\{\lambda_{n}^{2}\right\}, \cdots\left\{\lambda_{n}^{p}\right\}$ with $\lambda_{n}^{k}$ positive for $1 \leq k \leq p$ any $n$, and $\lim _{n \rightarrow \infty} \lambda_{n}^{k}=0$ for any $1 \leq k \leq p$, satisfying, for $j \neq k$,

$$
\frac{\lambda_{n}^{k}}{\lambda_{n}^{j}}+\frac{\lambda_{n}^{j}}{\lambda_{n}^{k}}+\frac{\left|a_{n}^{k}-a_{n}^{j}\right|^{2}}{\lambda_{n}^{k} \lambda_{n}^{j}} \rightarrow \infty, \text { as } n \rightarrow \infty,
$$

and

(c) a harmonic map $\omega_{0}$ from $S^{2}$ into $S^{N}$ and a sequence of non-constant harmonic maps $\left\{\omega_{k}\right\}_{k=1}^{p}$ from $S^{2}$ into $S^{N}$ with the properties

$$
u_{n}(x)-\sum_{k=1}^{p}\left(\omega_{k}\left(\frac{x-a_{n}^{k}}{\lambda_{n}^{k}}\right)-\omega_{k}(\infty)\right) \rightarrow \omega_{0}(x),
$$

strongly in $W_{l o c}^{1,2}\left(R^{2}, R^{K+1}\right)$ therefore

$$
\lim _{n \rightarrow \infty} \int_{B_{K}}\left|\nabla u_{n}\right|^{2} d x=\int_{B_{K}}\left|\nabla \omega_{0}\right|^{2} d x+\sum_{k=1}^{p} E\left(\omega_{k}\right) \text { for any } K<\infty .
$$

Remark 3. Possible bubbles drifting to infinity indeed could happen here as noticed by a referee. But Theorem 1.1 only concerns the local behavior of the solution of heat equation near a given singular point.

Note. In this paper, to simplify the exposition, we make no effort to distinguish sequences and subsequences: that is, we will often replace sequences with their subsequences and use the same indices.

Our proof of Proposition 1.2 is based on energy estimates over the necks connecting bubbles, together with a procedure for analyzing blow-ups which is similar to one used by Brezis and Coron in [4].

The author would like to thank professors Alice Chang and S.Y. Cheng for their constant encouragement and help. Moreover, the author is very grateful to Professors Michael Struwe and Paul Yang for many interesting conversations and their interests in this work. Also the author would like to thank Professor Itai Shafrir for pointing out some mistake in a previous version of this paper. And the author would like to thank Dr. Kevin Oden 
for his help in improving the writing of this paper. At last, but certainly not least, the author would like to express his heartful thanks to referees. Without their helpful suggestions we would not have this paper in the present form.

\section{Solutions of Heat Equations.}

In this section we shall study the local behavior of solutions of the heat equation for harmonic maps from surfaces near their singular points. First we will use the local energy comparison lemma from Struwe's paper [17] to prove the following:

Proposition 2.1. Suppose that $u$ is a solution of (1.1), and that $\left(x_{0}, T\right)$ and $B_{R}$ are given as in the statement of Theorem 1.1.

Then $\lim _{t \rightarrow T} \int_{B_{R}}|\nabla u|^{2}(\cdot, t) d x$ exists.

Proof. Suppose that

$$
\limsup _{t \rightarrow T} \int_{B_{R}}|\nabla u|^{2}(\cdot, t)=\lim _{t_{i} \rightarrow T} \int_{B_{R}}|\nabla u|^{2}\left(\cdot, t_{i}\right)=M+\int_{B_{R}}|\nabla u|^{2}(\cdot, T),
$$

and

$$
\liminf _{t \rightarrow T} \int_{B_{R}}|\nabla u|^{2}(\cdot, t)=\lim _{s_{i} \rightarrow T} \int_{B_{R}}|\nabla u|^{2}\left(\cdot, s_{i}\right)=m+\int_{B_{R}}|\nabla u|^{2}(\cdot, T) .
$$

Then to prove this proposition we only need to prove $m=M$. Take $\lambda_{j}=\frac{1}{j} R$. Since $u(x, t) \rightarrow u(x, T)$ strongly in $C^{\infty}$ on any compact subset of $\left.B_{R} \backslash\left\{x_{0}\right\}\right)$ as $t \rightarrow T$ (see Struwe's [17]), for each $j$ there exist $i_{j}$ such that

$$
\left.\int_{B_{R} \backslash B_{\lambda_{j}}\left(x_{0}\right)}|| \nabla u\left(\cdot, t_{i_{j}}\right)\right|^{2}-\left.|\nabla u(\cdot, T)|^{2}\left|d x<\frac{1}{2} \int_{B_{\lambda_{j}}\left(x_{0}\right)}\right| \nabla u(\cdot, T)\right|^{2} d x,
$$

and by (2.1),

$$
\begin{aligned}
\int_{B_{R}}\left|\nabla u\left(\cdot, t_{i_{j}}\right)\right|^{2} d x-\int_{B_{R}}|\nabla u(\cdot, T)|^{2} d x & \\
& \geq M-\frac{1}{2} \int_{B_{\lambda_{j}}\left(x_{0}\right)}|\nabla u(\cdot, T)|^{2} d x .
\end{aligned}
$$


Therefore we have

$$
\int_{B_{\lambda_{j}}\left(x_{0}\right)}\left|\nabla u\left(\cdot, t_{j}\right)\right|^{2} d x \geq M
$$

where $t_{j}=t_{i_{j}}$. Assume that,

$$
s_{j} \leq t_{j} \leq s_{j+1} \leq t_{j+1},
$$

by taking subsequences from $\left\{t_{j}\right\}$ and $\left\{s_{j}\right\}$ if necessary. For any $\delta>0$, by Lemma 3.6 in [17], we get

$$
\int_{B_{\delta}\left(x_{0}\right)}\left|\nabla u\left(\cdot, s_{j}\right)\right|^{2} d x \geq \int_{B_{\lambda_{j}}\left(x_{0}\right)}\left|\nabla u\left(\cdot, t_{j}\right)\right|^{2} d x-\frac{c\left|t_{j}-s_{j}\right|}{\left(\delta-\lambda_{j}\right)^{2}} E\left(u_{0}\right) .
$$

This implies

$$
\begin{array}{r}
\lim _{s_{j} \rightarrow T} \int_{B_{R}}|\nabla u|^{2}\left(\cdot, s_{j}\right)= \\
=\lim _{s_{j} \rightarrow T}\left\{\int_{B_{R} \backslash B_{\delta}\left(x_{0}\right)}\left|\nabla u\left(\cdot, s_{j}\right)\right|^{2}+\int_{B_{\delta}\left(x_{0}\right)}\left|\nabla u\left(\cdot, s_{j}\right)\right|^{2}\right\} \\
\geq M+\int_{B_{R} \backslash B_{\delta}\left(x_{0}\right)}|\nabla u(\cdot, T)|^{2} d x .
\end{array}
$$

Taking $\delta \rightarrow 0$, we can conclude that $m=M$, which completes the proof. To prove Theorem 1.1, we will choose the scaling as $\lambda_{j}$ defined in the above proof, and an appropriate time sequences to convert the problem into that of blow-ups of a Palais-Smale sequence. We then apply Proposition 1.2.

Proof of Theorem 1.1. By Proposition 2.1, we know there exists a number L such that

$$
\lim _{t \rightarrow T} \int_{B_{R}}|\nabla u|^{2}(\cdot, t)=L+\int_{B_{R}}|\nabla u|^{2}(\cdot, T),
$$

for $B_{R}$ given as in the statement of Theorem 1.1. Moreover, in the light of (2.5) there are sequences $\lambda_{j} \rightarrow 0, t_{j} \rightarrow T$ such that

$$
\int_{B_{\lambda_{j}}\left(x_{0}\right)}\left|\nabla u\left(\cdot, t_{j}\right)\right|^{2} d x \geq L .
$$


Now we do the scaling procedure by setting

$$
v_{j}(x, t)=u\left(\lambda_{j} x+x_{0},-\lambda_{j}^{2} t+t_{j}\right) .
$$

First of all,

$$
E\left(v_{j}(\cdot, t)\right)=E\left(u\left(\cdot,-\lambda_{j}^{2} t+t_{j}\right)\right) \leq E\left(u_{0}\right),
$$

for any $t \leq \frac{t_{j}}{\lambda_{j}^{2}}$. Secondly,

$$
\int_{0}^{S} \int_{\Omega}\left|\partial_{t} v_{j}\right|^{2} d x d t=\int_{t_{j}-\lambda_{j}^{2} S}^{t_{j}} \int_{\Omega_{j}}\left|\partial_{t} u\right|^{2} d x d t \rightarrow 0,
$$

as $j \rightarrow \infty$ for any given positive number $S$, where $\Omega$ is any bounded domain in $R^{2}$ and $\Omega_{j}=\lambda_{j} \Omega+x_{0}$. Because one has

$$
\begin{gathered}
\int_{t_{1}}^{t_{2}} \int_{B_{R}}\left|\partial_{t} u\right|^{2} \phi^{2} d x d t+\int_{t_{1}}^{t_{2}} \frac{d}{d t} \int_{B_{R}}|\nabla u|^{2} \phi^{2} d x d t \\
\leq C \int_{t_{1}}^{t_{2}} \int_{B_{r}}\left|\nabla u\left\|\partial_{t} u\right\| \nabla \phi\right| \phi d x d t,
\end{gathered}
$$

using $\partial_{t} u \phi^{2}$ as a testing function for equation (1.1), where $\phi \in C_{0}^{\infty}\left(B_{R}\right), \phi \equiv$ 1 in $B_{\frac{R}{2}}$ (see the proof of Lemma 3.6 in Struwe's paper [17]). Therefore

$$
\begin{aligned}
& \int_{t_{1}}^{t_{2}} \int_{B_{R}}\left|\partial_{t} u\right|^{2} d x d t \\
& \quad \leq \int_{B_{R}}|\nabla u|^{2}\left(\cdot, t_{1}\right) d x-\int_{B_{R}}|\nabla u|^{2}\left(\cdot, t_{2}\right) d x+C \frac{t_{2}-t_{1}}{R^{2}} \\
& \quad \leq C \sup _{\left[t_{1}, t_{2}\right]} \int_{B_{R} \backslash B_{\frac{R}{2}}}|\nabla u|^{2}(\cdot, t) d x+\int_{B_{\frac{R}{2}}}|\nabla u|^{2}\left(\cdot, t_{1}\right) d x \\
& \quad-\int_{B_{\frac{R}{2}}}|\nabla u|^{2}\left(\cdot, t_{2}\right) d x+C \frac{t_{2}-t_{1}}{R^{2}}
\end{aligned}
$$

Thus (2.11) is proved. Then for some number $\tau_{j} \in(0, S)$ we have

$$
E\left(v_{j}\right) \leq C, \text { and } \int_{\Omega}\left|\partial_{t} v_{j}\right|^{2} d x \rightarrow 0, \text { as } j \rightarrow \infty,
$$

where $v_{j}(x)=v_{j}\left(x, \tau_{j}\right)$. Now on one hand, for any positive number $K$,

$$
\int_{B_{K}}\left|\nabla v_{j}\right|^{2} d x=\int_{B_{\lambda_{j} K}}\left|\nabla u\left(\cdot,-\lambda_{j}^{2} \tau_{j}+t_{j}\right)\right|^{2} d x
$$


Applying Lemma 3.6 in [17] again, we have,

$$
\begin{aligned}
\int_{B_{\lambda_{j} K}}\left|\nabla u\left(\cdot,-\lambda_{j}^{2} \tau_{j}+t_{j}\right)\right|^{2} & \\
& \geq \int_{B_{\lambda_{j}}}\left|\nabla u\left(\cdot, t_{j}\right)\right|^{2} d x-\frac{c \tau_{j}}{(K-1)^{2}} E\left(u_{0}\right) .
\end{aligned}
$$

Therefore,

$$
\int_{B_{K}}\left|\nabla v_{j}\right|^{2} d x \geq L-\frac{c S}{(K-1)^{2}} E\left(u_{0}\right) .
$$

On the other hand, for any positive small number $\delta$,

$$
\int_{B_{\frac{\delta}{\lambda_{j}}}}\left|\nabla v_{j}\right|^{2} d x=\int_{B_{\delta}}\left|\nabla u\left(\cdot,-\lambda_{j}^{2} \tau_{j}+t_{j}\right)\right|^{2} d x .
$$

By the Proposition 2.1 we have,

$$
\lim _{j \rightarrow \infty} \int_{B_{\delta}}\left|\nabla u\left(\cdot,-\lambda_{j}^{2} \tau_{j}+t_{j}\right)\right|^{2} d x=L+\int_{B_{\delta}}|\nabla u(\cdot, T)|^{2} d x
$$

Thus

$$
\lim _{K \rightarrow \infty} \lim _{j \rightarrow \infty} \int_{B_{K}}\left|\nabla v_{j}\right|^{2} d x=L
$$

By the homogeneity of the scaling and (2.12), we can conclude that $\left\{v_{j}(x)\right\}$ is a Palais-Smale sequence to which we can apply Proposition 1.2. The only thing left is the convergence (1.4). In view of Proposition 1.2 we define

$$
f_{j}(x)=\omega_{0}(x)+\sum_{k=1}^{p}\left(\omega_{k}\left(\frac{x-a_{j}^{k}}{\lambda_{j}^{k}}\right)-\omega_{k}(\infty)\right),
$$

which has the property: $v_{j}(x)-f_{j}(x)$ strongly converges to zero in $W_{l o c}^{1,2}\left(R^{2}\right.$, $R^{K+1}$ ). So we have, for any given $K$ large enough,

$$
\begin{gathered}
\int_{B_{R}}\left|\nabla u\left(x, \widehat{t}_{j}\right)-\nabla f_{j}\left(\frac{x-x_{0}}{\lambda_{j}}\right)\right|^{2} d x=\int_{B_{\frac{R}{\lambda_{j}}}}\left|\nabla v_{j}-\nabla f_{j}\right|^{2} d x \\
=\int_{B_{\frac{R}{\lambda_{j}}} \backslash B_{K}}\left|\nabla v_{j}-\nabla f_{j}\right|^{2} d x+\int_{B_{K}}\left|\nabla v_{j}-\nabla f_{j}\right|^{2} d x \\
=\int_{B_{\frac{R}{\lambda_{j}}} \backslash B_{K}}\left|\nabla v_{j}-\nabla f_{j}\right|^{2} d x+o(1)
\end{gathered}
$$


where

$$
\begin{aligned}
& \int_{B_{\frac{R}{\lambda_{j}}} \backslash B_{K}}\left|\nabla v_{j}-\nabla f_{j}\right|^{2} d x \\
& =\int_{B_{\frac{R}{\lambda_{j}} \backslash B_{K}}}\left|\nabla v_{j}\right|^{2} d x+\int_{B_{\frac{R}{\lambda_{j}}} \backslash B_{K}}\left|\nabla f_{j}\right|^{2} d x-2 \int_{B_{\frac{R}{\lambda_{j}}} \backslash B_{K}} \nabla v_{j} \cdot \nabla f_{j} d x \\
& =\int_{B_{R} \backslash B_{\lambda_{j} K}}|\nabla u|^{2}\left(\cdot,-\lambda_{j}^{2} \tau_{j}+t_{j}\right) d x \\
& \quad+\int_{B_{\frac{R}{\lambda_{j}} \backslash B_{K}}}\left|\nabla f_{j}\right|^{2} d x-2 \int_{B_{\frac{R}{\lambda_{j}} \backslash B_{K}}} \nabla v_{j} \cdot \nabla f_{j} d x .
\end{aligned}
$$

By the definition of $\lambda_{j}$ in (16), it is easily seen that

$$
\int_{B_{R} \backslash B_{\lambda_{j} K}}|\nabla u|^{2}\left(\cdot,-\lambda_{j}^{2} \tau_{j}+t_{j}\right) d x=o(1)+\int_{B_{R}}|\nabla u|^{2}(\cdot, T) d x
$$

also,

$$
\begin{gathered}
\int_{B_{\frac{R}{\lambda_{j}}} \backslash B_{K}}\left|\nabla f_{j}\right|^{2} d x=o(1) \text { as } K \rightarrow \infty \\
\int_{B_{\frac{R}{\lambda_{j}}} \backslash B_{K}} \nabla v_{j} \cdot \nabla f_{j} d x=o(1), \text { as } K \rightarrow \infty
\end{gathered}
$$

since $f_{j}$ is a combination of the standard bubbles centered at given points $\left\{x^{k}\right\}$. Therefore

$$
\lim _{j \rightarrow \infty} \int_{B_{R}}\left|\nabla u\left(x,-\lambda_{j}^{2} \tau_{j}+t_{j}\right)-\nabla f_{j}\left(\frac{x-x_{0}}{\lambda_{j}}\right)\right|^{2} d x=\int_{B_{R}}|\nabla u|^{2}(\cdot, T) d x .
$$

On the other hand, again by the special form of $f_{j}$, we have

$$
\begin{aligned}
u\left(x, \widehat{t}_{j}\right)-\left(f_{j}\left(\frac{x-x_{0}}{\lambda_{j}}\right)-\omega_{0}(\infty)\right) & \rightarrow u(x, T) \\
& \text { as } j \rightarrow \infty \text { in } W^{1,2}\left(B_{R}, R^{K+1}\right) .
\end{aligned}
$$

Therefore we have the convergence (1.4). This finishes the proof of Theorem 1.1 . 


\section{Proof of Proposition 1.2.}

In this section we shall discuss the behavior of Palais-Smale sequences and prove Proposition 1.2. We all know that the deep regularity theory of minimizing harmonic maps due to Rick Schoen and Karen Uhlenbeck $[14,15]$ has had profound influences not only in the field of geometric analysis but also in the field of nonlinear partial differential equations. It also has been noticed that the recent works of Frederic Helein [10], Fabric Bethuel [1] and C.L.Evans [9] on the regularity of stationary harmonic maps captured important features of certain nonlinear differential equations by introducing Hardy space and Fefferman-Stein's duality theorem of BMO spaces and Hardy spaces to the nonlinear analysis. Helein first observed the special form of the equation for harmonic maps into spheres, though Yunmei Chen [8] and J. Shatah [16] seem to have been the first to deduce useful analytic consequences from this observation for weakly harmonic maps. We shall use the special form of the equation for harmonic maps into spheres in the proof of our key lemma in this section. Now let us state a lemma about $\epsilon$-compactness for a special kind of Palais-Smale sequences, whose proof is very standard in the light of Helein [10] and Qing [12].

Lemma 3.1. Suppose that $N$ is a compact manifold. And suppose that $u_{n} \in W^{1,2}\left(R^{2}, N\right)$ with

$$
-\Delta u_{n}=A\left(u_{n}\right)\left(\nabla u_{n}, \nabla u_{n}\right)+h_{n}
$$

where $h_{n}$ goes to zero in $L_{\text {loc }}^{2}\left(R^{2}, R^{k}\right)$, and that $u_{n} \rightarrow u$ in $W^{1,2}\left(R^{2}, R^{k}\right)$, $E\left(u_{n}\right) \leq C$. Then $u$ is a harmonic map. And, there exists an $\epsilon_{0}, u_{n}$ strongly converges to $u$ in $W^{1,2}\left(B_{R}, R^{k}\right)$ for any $r<R$ provided that $\int_{B_{R}}\left|\nabla u_{n}\right|^{2} d x \leq$ $\epsilon_{0}$.

Remark 4. About the harmonicity of the weak limit, in [2] Bethuel recently proved that a weak limit of any Palais-Smale sequence is still harmonic when the target is a general compact manifold.

Proof. First let us assume that $N$ has a global orthonormal frame on it without loss of any generality. Otherwise we extend the metric of $N$ to the tubular neighborhood $\tilde{N}$ of $N$ in $R^{K}$ so that $N$ is a totally geodesic submanifold of $\tilde{N}$ (see [10]), and we replace $N$ by $\tilde{N}$ if it is necessary. Note that the equation (3.1) stays the same since the image of $u_{n}$ lies in $N$. Then 
using the same notation in [12] (see Lemma 3 in [12]), there exists an $\epsilon_{0}$ such that

$$
\bar{\partial}\left(M^{i j}\left\langle\frac{\partial u}{\partial x}-\sqrt{-1} \frac{\partial u}{\partial y}, e_{i}\right\rangle\right)=\left\langle h, e_{j}\right\rangle \text { in } \Omega \subset R^{2},
$$

where $\left\{e_{i}\right\}$ is a global orthonormal frame on $N$ (we ignore the index $n$ here for a moment without confusion), and, as stated in Lemma 3 in [12], the norms of the metrix $\left(M_{i j}\right)$ and its inverse are bounded in $\Omega$ by a universal constant (independent on $n$ ) provided that $\int_{\Omega}|\nabla u|^{2} d x \leq \epsilon_{0}$. So by standard elliptic estimates and Sobolev Embedding Theorem we have $\epsilon$-compactriess since $\left\|\left\langle h, e_{j}\right\rangle\right\|_{L^{2}} \leq\|h\|_{L^{2}}$. Then, this lemma follows from the standard argument due to Sacks and Uhlenbeck [13]. So the proof is complete.

Next, we will state and prove a lemma which will be used to estimate the energy over the necks connecting bubbles. Let $\Omega=B_{R} \backslash B_{r}$ with $0<$ $r<R \leq 1$.

Lemma 3.2. Suppose that $u \in W^{1,2}\left(B_{R}, S^{K}\right)$ with $\int_{\Omega}|\nabla u|^{2} d x \leq \varepsilon$ satisfies

$$
\triangle u=u|\nabla u|^{2}+h \text { in } B_{R},
$$

where $\|h\|_{L^{2}\left(B_{R}\right)} \leq \varepsilon$. Assume that $v$ is given as follows

$$
\left\{\begin{array}{l}
\triangle v=0 \text { in } \Omega \\
\left.v\right|_{\partial \Omega}=\left.u\right|_{\partial \Omega}
\end{array}\right.
$$

Then there exists an $\varepsilon_{0}$ such that

$$
\int_{\Omega}|\nabla u|^{2} d x \leq C \int_{\Omega}|\nabla v|^{2} d x+C\|h\|_{L^{2}\left(B_{R}\right)}
$$

provided that $\varepsilon \leq \varepsilon_{0}$, where $C$ and $\varepsilon_{0}$ are constants independent on $\Omega$.

Proof. By the assumptions we have

$$
\begin{aligned}
-\triangle(u-v)^{k}= & \sum_{l, \alpha}\left\{\left(u^{k} u_{\alpha}^{l}-u^{l} u_{\alpha}^{k}\right) u_{\alpha}^{l}\right\}+h^{k} \\
= & \sum_{l, \alpha}\left\{\left(u^{k} u_{\alpha}^{l}-u^{l} u_{\alpha}^{k}\right)\left(u^{l}-v^{l}\right)_{\alpha}\right\} \\
& +\sum_{l, \alpha}\left\{\left(u^{k} u_{\alpha}^{l}-u^{l} u_{\alpha}^{k}\right) v_{\alpha}^{l}\right\}+h^{k} .
\end{aligned}
$$

Using the special algebraic structure we let $A^{k l}=\left(u^{k} u_{1}^{l}-u^{l} u_{1}^{k}, u^{k} u_{2}^{l}-u^{l} u_{2}^{k}\right)$. Then $\operatorname{div} A^{k l}=u^{l} h^{k}-u^{k} h^{l}$. Suppose we have $\phi^{k l}$ satisfying

$$
\left\{\begin{array}{l}
-\triangle \phi^{k l}=u^{k} h^{l}-u^{l} h^{k} \text { in } B_{R} \\
\left.\phi^{k l}\right|_{B_{R}}=0 .
\end{array}\right.
$$


Then we have $\operatorname{div}\left(A^{k l}-\nabla \phi^{k l}\right)=0$ in $B_{R}$. Next we rewrite the equation (3.6) into

$$
\begin{aligned}
-\Delta(u-v)^{k} & =\sum_{l}\left(A^{k l}-\nabla \phi^{k l}\right) \nabla(u-v)^{l} \\
& +\operatorname{div}\left(\sum_{l} \nabla \phi^{k l}(u-v)^{l}\right)-\sum_{l} \Delta \phi^{k l}(u-v)^{l} \\
& +\sum_{l} A^{k l} \cdot \nabla v^{l}+h^{k} .
\end{aligned}
$$

Now let $w_{1}$ satisfy

$$
\left\{\begin{array}{l}
-\triangle w_{1}^{k}=\sum_{l}\left(A^{k l}-\nabla \phi^{k l}\right) \nabla(u-v)^{l} \quad \text { in } \Omega \\
\left.w_{1}^{k}\right|_{\partial \Omega}=0,
\end{array}\right.
$$

and let $w_{2}$ satisfy

$$
\left\{\begin{array}{l}
-\Delta w_{2}^{k}=\operatorname{div}\left(\sum_{l} \nabla \phi^{k l}(u-v)^{l}\right) \text { in } \Omega \\
\left.w_{2}^{k}\right|_{\partial \Omega}=0 .
\end{array}\right.
$$

To estimate $w_{1}$, we notice that $\operatorname{div}\left(A^{k l}-\nabla \phi^{k l}\right)=0$ in $B_{R}$. By Theorem 1.2 in [3] (see also [7] of S.Chanillo and Yanyan Li) we have

$$
\left\|\nabla w_{1}\right\|_{L^{2}(\Omega)} \leq C \sum_{k, l}\left\|A^{k l}-\nabla \phi^{k l}\right\|_{L^{2}(\Omega)}\left\|\nabla(u-v)^{l}\right\|_{L^{2}(\Omega)},
$$

where $C$ is independent of $\Omega$. The key point here is that, by the fact that $B_{R}$ is simply connected, we can rewrite the equation into a form to which Theorem 1.2 in [3] can be applied. And to estimate $w_{2}$, we simply multiply two sides of the equation (3.10) by $w_{2}$ and integrate over $\Omega$

$$
\begin{aligned}
\left\|\nabla w_{2}\right\|_{L^{2}(\Omega)} & \leq \sum_{k}\left\|\sum_{l} \nabla \phi^{k l}(u-v)^{l}\right\|_{L^{2}(\Omega)} \\
& \leq\|\nabla \phi\|_{L^{4}\left(B_{R}\right)}\|u-v\|_{L^{4}(\Omega)} \\
& \leq C\|h\|_{L^{2}\left(B_{R}\right)}\|\nabla(u-v)\|_{L^{2}(\Omega)}
\end{aligned}
$$


where again $C$ is independent of $\Omega$. Now we multiply the equation (3.8) by $u-v$ and integrate over $\Omega$. We get

$$
\begin{gathered}
\int_{\Omega}|\nabla(u-v)|^{2} d x=\sum_{k}\left\{\int_{\Omega}\left(-\Delta w_{1}^{k}\right)(u-v)^{k} d x \int_{\Omega}\left(-\Delta w_{2}^{k}\right)(u-v)^{k} d x\right\} \\
+\sum_{k, l}\left\{\int_{\Omega}\left(u^{k} h^{l}-u^{l} h^{k}\right)(u-v)^{k}(u-v)^{l} d x+\int_{\Omega} A^{k l} \nabla v^{l}(u-v)^{k} d x\right\} \\
\quad+\sum_{k} \int_{\Omega} h^{k}(u-v)^{k} d x . \\
\leq C \epsilon \int_{\Omega}|\nabla(u-v)|^{2} d x+C \int_{\Omega}|\nabla u||\nabla v| d x+C\|h\|_{L^{2}\left(B_{R}\right) \cdot}
\end{gathered}
$$

Since all $C^{\prime}$ s here are independent of $\Omega$, when $\varepsilon$ is small enough we have the energy control for $u-v$ as follows:

$$
\int_{\Omega}|\nabla(u-v)|^{2} d x \leq c \int_{\Omega}\left|\nabla u\|\nabla v \mid d x+c\| h \|_{L^{2}\left(B_{R}\right)} .\right.
$$

This implies this lemma in view of the following Schwarz inequality

$$
\int_{\Omega}|\nabla u|^{2} d x \leq 2 \int_{\Omega}|\nabla(u-v)|^{2} d x+2 \int_{\Omega}|\nabla v|^{2} d x .
$$

Thus the proof is completed.

Remark 5. In the proof of the above lemma, the use of Theorem 1.2 in [3] seems very crucial. The independence of $C$ on the domain is understood as a special feature of the equations studied in [2] and [7].

By Lemma 3.1, which is the so-called $\varepsilon$-compactness lemma, through a standard argument we know that for $u_{n}$ and $u$ as given in Lemma 3.1, there exist at most finitely many points $x^{1}, x^{2}, \cdots, x^{l}$ such that $u_{n}$ converges to $u$ strongly in $W_{l o c}^{1,2}\left(R^{2} \backslash\left\{x^{1}, x^{2}, \cdots, x^{l}\right\}, S^{K}\right)$. So what we are really concerned with is the local behavior of $u_{n}$ around each of these special points. For simplicity, we will focus our attention on a single blow-up point $\bar{x} \in\left\{x^{k}\right\}_{k=1}^{l}$. Let

$$
B_{\delta}(\bar{x}) \bigcap\left(\left\{x^{k}\right\}_{k=1}^{l} \backslash \bar{x}\right)=\phi .
$$

Now we employ the quantity $Q_{n}(t)$ used by Brezis and Coron in [4] to detect bubbles. Namely,

$$
Q_{n}(t)=\sup _{x \in B_{\delta}(\bar{x})} \int_{x+t D}\left|\nabla u_{n}\right|^{2} d x
$$


where $D$ is the unit disk in $R^{2}$. Then for $\varepsilon_{0}$ given in Lemma 3.1 and Lemma 3.2 we can find $\lambda_{n}$ and $a_{n}$ such that

$$
\int_{a_{n}+\lambda_{n} D}\left|\nabla u_{n}\right|^{2} d x=Q_{n}\left(\lambda_{n}\right)=\frac{\varepsilon_{0}}{2} .
$$

It is clear that $\inf _{n \rightarrow \infty} \lambda_{n}=0$ and $\lim _{n \rightarrow \infty} a_{n}=\bar{x}$, otherwise $\bar{x}$ would not be a blow-up point by Lemma 3.1.

Proof of Proposition 1.2. It is quite easy to take out the first bubble by scaling with $\lambda_{n}$ and $a_{n}$, namely let $\widetilde{u}_{n}(x)=u_{n}\left(\lambda_{n} x+a_{n}\right)$. Then $\widetilde{u}$ satisfies

$$
\triangle \widetilde{u}_{n}=A\left(\widetilde{u}_{n}\right)\left(\nabla \widetilde{u}_{n}, \nabla \widetilde{u}_{n}\right)+\lambda_{n}^{2} h_{n}\left(\lambda_{n} x+a_{n}\right) .
$$

It is easy to see that $\lambda_{n}^{2} h_{n}\left(\lambda_{n} x+a_{n}\right) \rightarrow 0$ in $L^{2}$. Therefore by Lemma 3.1 we know $\widetilde{u}_{n} \rightarrow \omega$ strongly in $W_{l o c}^{1,2}\left(R^{2}, R^{K+1}\right)$, where $\omega$ is a non-constant harmonic map from $S^{2}$ into $S^{K}$ since $\int_{D}|\nabla \omega|^{2} d x=\frac{\varepsilon_{0}}{2}>0$. Moreover, if we let

$$
\omega_{0}=u, \lambda_{n}^{1}=\lambda_{n}, a_{n}^{1}=a_{n} \text { and } \omega_{1}=\omega,
$$

we have

$$
\begin{aligned}
\int_{B_{\delta}}\left|\nabla\left(u_{n}(x)-\omega_{1}\left(\frac{x-a_{n}^{1}}{\lambda_{n}^{1}}\right)\right)\right|^{2} d x \\
=\int_{B_{\delta}}\left|\nabla u_{n}\right|^{2} d x-\int_{R^{2}}\left|\nabla \omega_{1}\right|^{2} d x+o(1)
\end{aligned}
$$

and

$$
u_{n}(x)-\left[\omega_{1}\left(\frac{x-a_{n}^{1}}{\lambda_{n}^{1}}\right)-\omega_{1}(\infty)\right] \rightarrow \omega_{0}(x),
$$

in $W^{-1,2}\left(R^{2}, R^{K+1}\right)$. Now we are going to detect the second bubble. As we said in Note 1, we may assume that $\lim _{n \rightarrow \infty} \int_{B_{\delta}}\left|\nabla u_{n}\right|^{2} d x$ exists. By (3.21) we know that

$$
\int_{B_{\delta}}\left|\nabla \omega_{0}\right|^{2} d x \leq \lim _{n \rightarrow \infty} \int_{B_{\delta}}\left|\nabla u_{n}\right|^{2} d x-\int_{R^{2}}\left|\nabla \omega_{1}\right|^{2} d x
$$

and equality holds if and only if

$$
u_{n}(x)-\left[\omega_{1}\left(\frac{x-a_{n}^{1}}{\lambda_{n}^{1}}\right)-\omega_{1}(\infty)\right] \rightarrow \omega_{0}(x)
$$


strongly in $W_{l o c}^{1,2}\left(R^{2}, R^{K+1}\right)$. If this happens, we can stop searching for bubbles, and Proposition 1.2 holds. Otherwise, we have

$$
\int_{B_{\delta}}\left|\nabla \omega_{0}\right|^{2} d x<\lim _{n \rightarrow \infty} \int_{B_{\delta}}\left|\nabla\left(u_{n}-\omega_{1}\left(\frac{x-a_{n}^{1}}{\lambda_{n}^{1}}\right)\right)\right|^{2} d x
$$

which implies

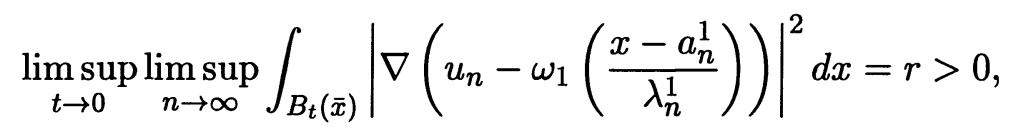

since $\bar{x}$ is an isolated blow-up point. Now let $v_{n}(x)=u_{n}(x)-\left(\omega_{1}\left(\frac{x-a_{n}^{1}}{\lambda_{n}^{1}}\right)-\right.$ $\left.\omega_{1}(\infty)\right)$ and redefine $Q_{n}(t)$ as follows:

$$
Q_{n}(t)=\sup _{x \in B_{\delta}} \int_{x+t D}\left|\nabla v_{n}\right|^{2} d x .
$$

By (3.25) again we can find $\lambda_{n}$ and $a_{n}$ such that

$$
\int_{a_{n}+\lambda_{n} D}\left|\nabla v_{n}\right|^{2} d x=Q_{n}\left(\lambda_{n}\right)=\min \left(\frac{r}{2}, \frac{\varepsilon_{0}}{2}\right)>0,
$$

and

$$
\lim _{n \rightarrow \infty} a_{n}=\bar{x}, \lim _{n \rightarrow \infty} \lambda_{n}=0 .
$$

Next we are going to prove that

$$
\frac{\lambda_{n}}{\lambda_{n}^{1}}+\frac{\left|a_{n}-a_{n}^{1}\right|}{\lambda_{n}^{1}} \rightarrow \infty \text { as } n \rightarrow \infty
$$

by contradiction. In fact, if $\frac{\lambda_{n}}{\lambda_{n}^{1}}<M$ and $\frac{\left|a_{n}-a_{n}^{1}\right|}{\lambda_{n}^{1}}<M$ for some big number $M$, then

$$
\begin{aligned}
\int_{a_{n}+\lambda_{n} D} & \left|\nabla v_{n}\right|^{2} d x \\
& \leq \int_{a_{n}^{1}+3 M \lambda_{n}^{1} D}\left|\nabla v_{n}\right|^{2} d x=\int_{3 M D}\left|\nabla\left(\widetilde{u}_{n}-\omega_{1}\right)\right|^{2} d x=o(1)
\end{aligned}
$$


because $\widetilde{u}_{n} \rightarrow \omega$ strongly in $W_{\text {loc }}^{1,2}\left(R^{2}, R^{K+1}\right)$. This is obviously contradicts the definition of $a_{n}$ and $\lambda_{n}$. Now we do the scaling procedure again for $v_{n}$ with $a_{n}$ and $\lambda_{n}$, which yields

$$
\widetilde{v}_{n}(x)=u_{n}\left(\lambda_{n} x+a_{n}\right)-\left[\omega_{1}\left(\frac{\lambda_{n}}{\lambda_{n}^{1}} x+\frac{a_{n}-a_{n}^{1}}{\lambda_{n}^{1}}\right)-\omega_{1}(\infty)\right] \rightarrow \omega(x),
$$

for some $\omega$. By (3.29) it is not hard to see that $\omega_{1}\left(\frac{\lambda_{n}}{\lambda_{n}^{1}} x+\frac{a_{n}-a_{n}^{1}}{\lambda_{n}^{1}}\right)-\omega_{1}(\infty) \rightarrow 0$. Therefore $\omega$ is a harmonic map from $S^{2}$ into $S^{K}$ by Lemma 3.1. What we need now is to prove that $\widetilde{v}_{n} \rightarrow \omega$ strongly in $W_{l o c}^{1,2}\left(R^{2}, R^{K+1}\right)$, which will imply that $\omega$ is not a constant map by (3.27). To do so, we consider two different cases.

$\underline{\text { Case } 1}$

$$
\frac{\lambda_{n}}{\lambda_{n}^{1}}<M, \frac{\left|a_{n}-a_{n}^{1}\right|}{\lambda_{n}^{1}} \rightarrow \infty ; \text { or } \frac{\lambda_{n}}{\lambda_{n}^{1}} \rightarrow \infty, \frac{\left|a_{n}-a_{n}^{1}\right|}{\lambda_{n}} \rightarrow \infty .
$$

In fact this is the case of joint bubbles (see Figure 1), since they are essentially separated. In other words, given any big number $L$ we have

$$
\left(a_{n}+L \lambda_{n} D\right) \bigcap\left(a_{n}^{1}+L \lambda_{n}^{1} D\right)=\phi,
$$

for $n$ large enough. Therefore $\omega_{1}\left(\frac{\lambda_{n}}{\lambda_{n}^{1}} x+\frac{a_{n}-a_{n}^{1}}{\lambda_{n}^{1}}\right) \rightarrow \omega_{1}(\infty)$ in $W_{l o c}^{1,2}\left(R^{2}, R^{K+1}\right)$. Then by Lemma 3.1 with (3.27) (apply Lemma 3.1 to $u_{n}\left(\lambda_{n} x+a_{n}\right)$ ) and some covering lemma we can conclude that $\widetilde{v}_{n} \rightarrow \omega$ strongly in $W_{l o c}^{1,2}\left(R^{2}, R^{K+1}\right)$.

Before we go to the second case, we need to prove a key lemma derived from Lemma 3.2 as follows:

Lemma 3.3. Suppose that $u$ and $\Omega$ are given as in Lemma 3.2, and that

$$
|u-P|_{W^{\frac{1}{2}, 2}\left(\partial B_{R}, R^{K+1}\right)}<\delta,|u-Q|_{W^{\frac{1}{2}, 2}\left(\partial B_{R}, R^{K+1}\right)}<\delta,
$$

for some points $P, Q \in S^{K}$. Then

$$
\int_{\Omega}|\nabla u|^{2} d x \leq C \delta+C \frac{|P-Q|^{2}}{\log \frac{R}{r}}+C\|h\|_{L^{2}\left(B_{R}\right)},
$$

where $C^{\prime}$ s are independent on $\Omega$. 


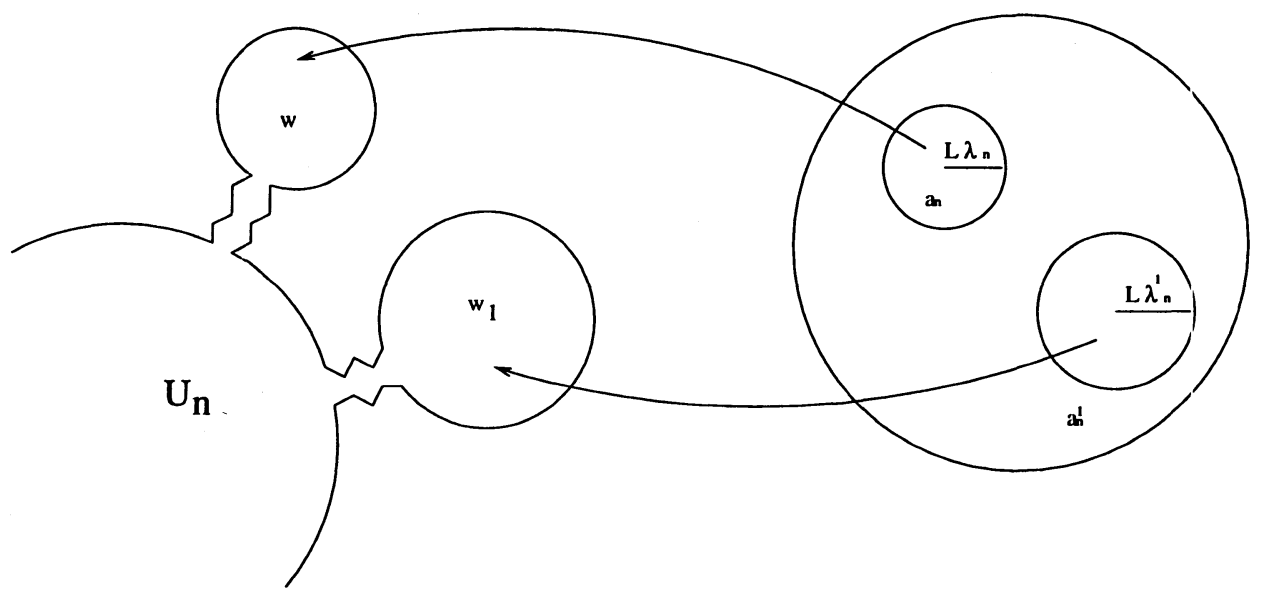

Figure 1.

Proof. Since we can explicitly solve the equations:

$$
\left\{\begin{array}{l}
\Delta w=0 \\
\left.w\right|_{\partial B_{R}}=P,\left.w\right|_{\partial B_{R}}=Q,
\end{array}\right.
$$

and compute the energy as in the paper [12]. Namely,

$$
\int_{\Omega}|\nabla w|^{2} d x=2 \pi \frac{|P-Q|^{2}}{\log \frac{R}{r}} .
$$

It is easy to get the result from Lemma 3.2 since

$$
\int_{\Omega}|\nabla v|^{2} \leq C \delta+\int_{\Omega}|\nabla w|^{2}
$$

Now we proceed to the second case.

$\underline{\text { Case } 2}$

$$
\frac{\lambda_{n}}{\lambda_{n}^{1}} \rightarrow \infty \text { and } \frac{\left|a_{n}-a_{n}^{1}\right|}{\lambda_{n}}<M .
$$

This is the case of bubbles over bubbles (see Figure 2). We need to control the energy over the necks connecting bubbles. Using Lemma 3.1 as in the first case we notice that, first, $u_{n}\left(\lambda_{n} x+a_{n}\right)$ converges to $\omega(x)$ strongly in $W_{l o c}^{1,2}\left(R^{2} \backslash B_{\epsilon}\left(\frac{a_{n}^{1}-a_{n}}{\lambda_{n}}\right)\right)$ for any small given $\epsilon$; second, $u_{n}\left(\lambda_{n}^{1} x+a_{n}^{1}\right)$ converges 


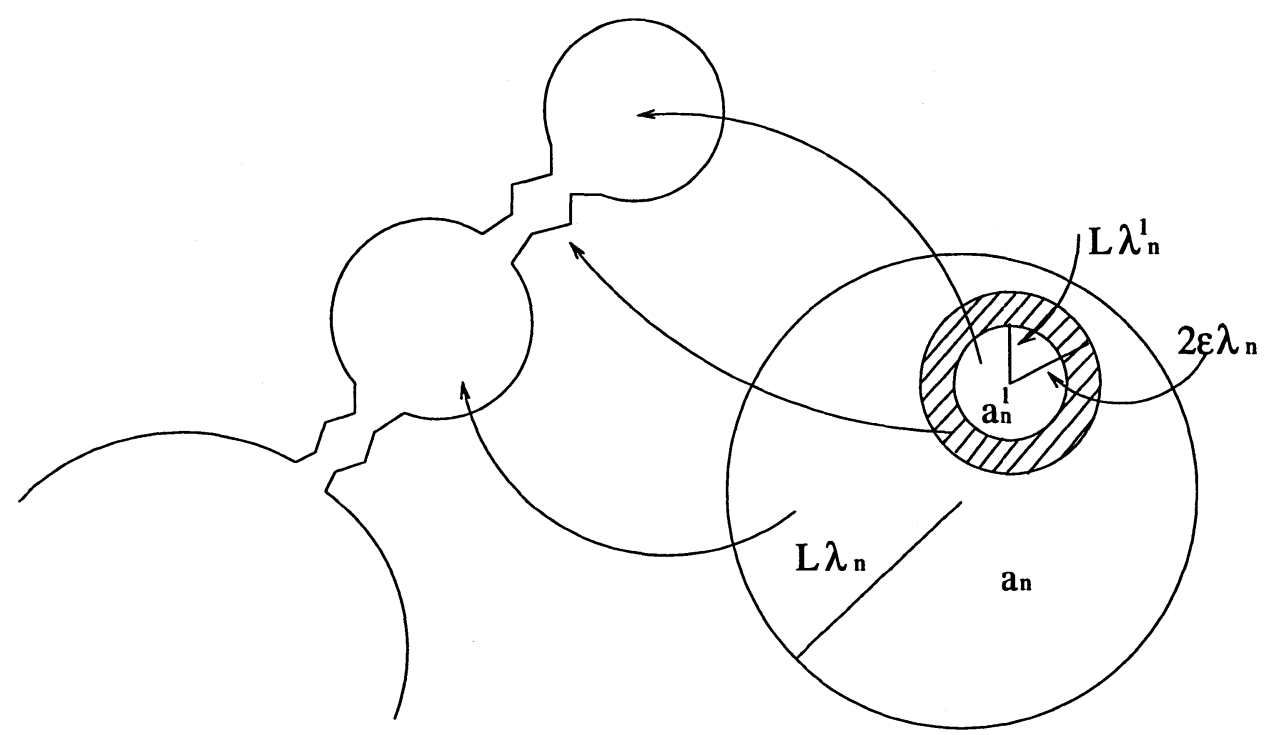

Figure 2.

to $\omega_{1}$ strongly in $W^{1,2}\left(B_{K}, R^{K+1}\right)$ for any given large $K$. Therefore, for any large number $L$

$$
\begin{aligned}
& \int_{B_{L}(0)}\left|\nabla\left(\widetilde{v}_{n}-\omega\right)\right|^{2} d x \\
& \leq \int_{B_{2 \epsilon}\left(\frac{a_{n}^{1}-a_{n}}{\lambda_{n}}\right)}\left|\nabla\left(\widetilde{v}_{n}-\omega\right)\right|^{2} d x+o(1) \\
& \leq \int_{B_{2 \epsilon \lambda_{n}}\left(a_{n}^{1}\right) \backslash B_{K \lambda_{n}^{1}}\left(a_{n}^{1}\right)}\left|\nabla u_{n}\right|^{2} d x+o(1) .
\end{aligned}
$$

Now we need to estimate the energy over the circular annulus $B_{2 \epsilon \lambda_{n}}\left(a_{n}^{1}\right) \backslash$ $B_{K \lambda_{n}^{1}}\left(a_{n}^{1}\right)$. It is not hard to see that

$$
\left|u_{n}-\omega(P)\right|_{W^{\frac{1}{2}, 2}\left(\partial B_{2 \epsilon \lambda_{n}}\left(a_{n}^{1}\right), R^{K+1}\right)}<o(1),
$$

where $P$ is the limit of $\frac{1}{\lambda_{n}}\left(a_{n}-a_{n}^{1}\right)$ and

$$
\left|u_{n}-\omega_{1}(\infty)\right|_{W^{\frac{1}{2}, 2}\left(\partial B_{K \lambda_{n}^{1}}\left(a_{n}^{1}\right), R^{K+1}\right)}<o(1) .
$$

This follows from the strong $W^{1,2}$-convergence away from this circular annulus. Moreover by (3.27)

$$
\int_{B_{2 \epsilon \lambda_{n}}\left(a_{n}^{1}\right) \backslash B_{K \lambda_{n}^{1}}\left(a_{n}^{1}\right)}\left|\nabla u_{n}\right|^{2} d x<\varepsilon_{0},
$$


when $\epsilon$ is small enough and $K$ is large enough. So using Lemma 3.3 and the fact that $\frac{\lambda_{n}}{\lambda_{n}^{1}} \rightarrow \infty$ we can conclude that $\widetilde{v}_{n} \rightarrow \omega$ strongly in $W_{l o c}^{1,2}$. To finish the proof of Proposition 1.2 we only need to do the above procedure of searching for bubbles over and over until

$$
\int_{B_{\delta}}\left|\nabla \omega_{0}\right|^{2} d x=\lim _{n \rightarrow \infty} \int_{B_{\delta}}\left|\nabla u_{n}\right|^{2} d x-\sum_{k=1}^{p} \int_{R^{2}}\left|\nabla \omega_{k}\right|^{2} d x .
$$

This implies

$$
u_{n}-\sum_{k=1}^{p}\left(\omega_{k}\left(\frac{x-a_{n}^{k}}{\lambda_{n}^{k}}\right)-\omega_{k}(\infty)\right) \rightarrow \omega_{0},
$$

strongly in $W_{l o c}^{1,2}\left(R^{2}, R^{K+1}\right)$. Thus the proof is complete.

Remark 6. From the works of Sacks and Uhlenbeck, Schoen and Uhlenbeck $[13,14,15]$, it is not difficult to see that Proposition 1.2 holds if the domain is any compact surface equipped with a given metric with or without boundary. Let us be reminded that Theorem 1.2 in [3] (Theorem 0.2 in [7]) is valid not only for the laplacian of $R^{2}$ but also for a much larger class of elliptic operators including the laplacians of any surfaces with a given metric. For the case when the domain has a boundary we recall the argument in Chang's paper [5], which essentially says that all blow-ups occur in the interior in the following sense,

$$
\frac{\operatorname{dist}\left(a_{k}^{j}, \partial \Omega\right)}{\lambda_{k}^{j}} \rightarrow \infty \text { as } j \rightarrow \infty .
$$

\section{References.}

[1] F.Bethuel, On the singular set of stationary harmonic maps, Manuscripta Math. 78 (1993), 417-443.

[2] F.Bethuel, Weak limit of Palais-Smale sequence for a class of critical functionals, Preprint.

[3] F.Bethuel and J.M.Ghidaglia, Improved regularity of solutions to elliptic equations involving Jacobians and applications, to appear in J. Maths pures et appliquées. 
[4] H.Brezis and J.M.Coron, Convergence of solutions of H-systems or how to blow bubbles, Archive Rat.Mech.Anal. 89 (1985), 21-56.

[5] K.C.Chang, Heat flow and boundary value problem for harmonic maps, Ann Inst. Henri Poincaré, Analyse non linéaire, Vol 6, No.5 (1989), 363-395.

[6] K.C.Chang, W.Ding and R.Ye, Finite time blow-up of the heat flow of harmonic maps from surfaces, J. Diff. Geo. 36 (1992), 507-515.

[7] S.Chanillo and Y.Li, Continuity of Solutions of uniformly elliptic equations in $R^{2}$, Manuscripta Math. 77 (1992), 415-433.

[8] Y.M.Chen, Weak solutions to the evolution problem for harmonic maps, Math. Zeit. 201 (1989), 69-74.

[9] C.L.Evans, Partial regularity for stationary harmonic maps into spheres, Arch. Rat. Mech.Anal. 116 (1991), 101-113.

[10] F.Helein, Régularité des applications faiblement harmoniques entre une surface at une varieté Riemannienne, C.R.Acad.Sci. Paris, t.312, Série I (1991), 591-596.

[11] J.Jost, "Two-dimensional geometric variational problems." New York, Wiley, 1991.

[12] J.Qing, Boundary regularity of weakly harmonic maps from surfaces, J. Funct. Anal. 114 (1993), 458-466.

[13] J.Sacks and K.Uhlenbeck. The Existence of Minimal Immersions of 2-spheres. Annals of Math. 113(81) pp1-24.

[14] R.Schoen and K.Uhlenbeck, Regularity Theory for Harmonic Maps, J. Diff. Geom. 17 (1982), 307-335.

[15] R.Schoen and K.Uhlenbeck, Boundary Regularity and the Dirichlet Problem for Harmonic Maps, J. Diff. Geom. 18 (1983), 253-268.

[16] J.Shatah, Weak solutions and development of singularities of the $\mathrm{SU}(2) \sigma$ model, Comm. Pure Appl. Math. 41 (1988), 459-469.

[17] M.Struwe, On the evolution of harmonic mappings of Riemannian surfaces, Comment. Math. Helvetici 60 (1985), 558-581.

JIE QING

DePartment of MATHEMATICS

Columbia University

NEW YORK, NY 10027

ReCEIVEd MaRCH 31st, 1995. 